\title{
Penser la primauté du masculin - sémantique du genre grammatical, perspectives synchroniques et diachroniques
}

\author{
Lucy Michel $^{\mathrm{a}}$ \\ Université de Bourgogne (GReLiSC - EA 4178 CPTC), 2 Boulevard Gabriel, 21000 Dijon, France
}

\begin{abstract}
Résumé. Le genre grammatical demeure en langue française un outil mystérieux, à la fois répartitoire des substantifs et phénomène d'accord, inhérent au substantif mais pouvant parfois varier, arbitraire ou motivé, et surtout, bipartite mais asymétrique. De fait, les descriptions et pratiques du genre grammatical en français s'appuient massivement sur l'adage (discuté ou non): «le masculin l'emporte sur le féminin»-comme forme première, genre par défaut et héritier privilégié du neutre latin. C'est cette primauté que nous tâcherons de questionner en synchronie comme en diachronie, en tenant compte des mécanismes intra- et extra-linguistiques en jeu de la construction de celle-ci. Nous centrerons surtout notre développement sur la classe des «dénominations de la personne » variables en genre. En effet, c'est principalement cette classe de noms qui alimente la réflexion sur l'émergence et le fonctionnement sémanticoréférentiel du genre grammatical. L'étude que nous en proposons s'appuie sur un ensemble d'ouvrages grammaticaux et lexicographiques modernes (XVI-XVIIIème) et contemporains (XIX-XXIème), et vise à la fois à discuter la pertinence des croyances associées au masculin en langue française, et à proposer une description sémantique du genre grammatical qui puisse problématiser le rapport supposément univoque entre sexe et genre des noms d'humains.
\end{abstract}

\begin{abstract}
The primacy of the masculine gender - a semantic reflection on grammatical gender, synchrony and diachrony. In French, grammatical gender still seems mysterious: it is both a classification tool and a grammatical agreement system, inherent to the noun but possibly variable, arbitrary or motivated, and above all, dual but asymmetrical. Most descriptions and usages of grammatical gender are
\end{abstract}

\footnotetext{
a Auteur de correspondance : lucy.michel@u-bourgogne.fr
} 


\begin{abstract}
founded on the phrase : "the masculine prevails over the feminine » - the masculine is considered as the true form of the noun, the choice by default and the main inheritor to the Latin neuter. It is precisely this prevailance that we will try to question, both synchronically and diachronically, by focusing on the linguistic mecanisms at play in its construction. We will mainly concentrate on variable "person denomination " nouns. This type of noun is indeed crucial in the study of grammatical gender, its origins and its semantico-referential functioning. The following development is based on several French grammatical studies and dictionaries from the XVIth to the XXIst century : we will both discuss the beliefs that surround the masculine gender within the French language, and try to propose a semantic description of the grammatical gender that questions the preconception of a univocal link between the sex of the referent and the gender of its denomination.
\end{abstract}

\title{
Introduction
}

Quel que soit l'angle d'attaque, un des constats qui émergent le plus rapidement dans les réflexions sur le genre grammatical est celui d'un fonctionnement double de la catégorie, différencié selon le type de référent. Ce constat conduit à l'idée d'une véritable rupture au sein de ladite catégorie, entre noms désignant des inanimés et des animés nonanthropomorphisés ${ }^{1}$, et noms d'animés humains ou anthropomorphisés. On aurait donc d'un côté un fonctionnement largement considéré comme arbitraire, qui fait du genre grammatical un pur outil de cohérence textuelle (par l'accord ou la reprise anaphorique) et d'opposition systémique entre masculin et féminin. C'est la position tenue entre autres par de nombreux linguistes structuralistes, et défendue par des auteurs comme A. Martinet, qui affirme que «l'information qu'apporte aux usagers de la langue l'existence des genres féminin et masculin est pratiquement nulle » (Martinet 1996, p. 218). De l'autre côté, on retrouve l'idée d'un lien motivé entre certains critères extra-linguistiques (ici, le sexe du référent) et la répartition des substantifs entre genre grammatical féminin et genre grammatical masculin. C'est la position la plus courante, celle qui est fondée sur le référentialisme, et qu'on retrouve dans la plupart des grammaires descriptives actuelles. Cette deuxième classe de substantifs, celle des noms d'humains $(\mathrm{NH})^{2}$, est au centre des discours, spécialisés ou non, sur la catégorie du genre grammatical. Plus précisément, l'intérêt se porte généralement sur les noms dits "variables en genre », qui ont un socle morphosyntaxique commun mais dont le genre grammatical peut varier en fonction du sexe du référent (ex. boucher, bouchère ; infirmier, infirmière ; con, conne ; etc.). Tous ces noms variables relèvent en fait de ce que nous nommons la «dénomination de la personne ». Sous cette étiquette, nous rangeons tous les substantifs pouvant permettre de dénommer un être humain singulier, et dont le sens n’est pas indéfini.

Cette définition permet d'exclure des substantifs comme foule, groupe, ensemble, qui renvoient à des entités plurielles, ou encore personne, individu, de sens indéfini, et de se concentrer sur une classe de noms plus précise, et au fonctionnement plus homogène. On constate en effet que les polémiques autour du genre grammatical concernent généralement (sans que cela soit exclusif) le domaine de la dénomination de la personne. Autour de cette classe de noms, un grand nombre de questions émergent: comment nommer indifféremment au sexe ? Quelle est la forme première des mots variables ? Le genre 
grammatical, au-delà de sa référence au sexe, a-t-il un sens ? À ces questions qui interrogent à la fois les grilles théoriques de lecture de la catégorie et les pratiques et usages que nous en faisons, la tradition grammaticale répond largement par la centralité du genre grammatical masculin, car comme le rappelle $\mathrm{A}$. Meillet: "le thème masculin est le principal [...]. Le genre féminin apparaît ainsi comme un sous-genre à l'intérieur du genre animé » (Meillet 1921, p. 213).

Afin de comprendre cette centralité, d'en révéler les enjeux, nous confronterons dans cette étude approches synchronique et diachronique du genre grammatical en langue française, dans le cadre de la dénomination de la personne, telle que définie ci-dessus. Nous partirons donc des descriptions grammaticales actuelles de la catégorie (1), en nous attardant sur la façon dont la primauté d'un genre grammatical par rapport à l'autre est exposée et validée. Nous tenterons ensuite de comprendre les mécanismes de construction et de stabilisation de ces procédés à partir des XVIème et XVIIème siècles (2), pour, à terme, en discuter la pertinence et la nécessité. Nous tâcherons enfin de proposer (3) un modèle de lecture du genre grammatical appuyé sur un questionnement culturel et historique plus explicite, ainsi que sur une critique constructiviste de cette catégorie linguistique.

\section{Du masculin partout, tout le temps}

\subsection{Les trois piliers de la primauté du masculin}

La tradition grammaticale française ${ }^{3}$ est marquée par une forte croyance en la prédominance du genre grammatical masculin sur le genre grammatical féminin. Celle-ci est fermement ancrée dans les pratiques et représentations de la langue française, et résumée, comme le rappelle Éliane Viennot tout au long de son récent ouvrage sur la question (Viennot 2014), par l'adage souvent sans source explicite « le masculin l'emporte sur le féminin ». Mais cet adage, quelque généralisé qu'il soit, s’appuie sur des faits linguistiques précis, qui constituent les trois piliers fondant la primauté du genre grammatical masculin :

1. l'accord au masculin: lorsque deux substantifs de genre différent sont caractérisés par un même adjectif, celui-ci s’accorde au masculin pluriel ;

2. le masculin forme première : la forme féminine des noms variables en genre est construite à partir de la forme masculine ;

3. la « valeur générique » du masculin : le masculin permet de dénommer, dans le cadre de la dénomination de la personne, indifféremment au sexe. ${ }^{4}$

Une des raisons pour lesquelles ces phénomènes ne viennent pas toujours d'un bloc à l'esprit est qu’ils sont généralement traités séparément. Et c’est précisément en les considérant comme des faits isolés que les ouvrages linguistiques et/ou lexicographiques contribuent à les asseoir comme règles indubitables, purement linguistiques, et surtout, structurellement nécessaires au bon fonctionnement de la langue. C'est en effet l'impression qui ressort de la lecture de quelques grammaires descriptives, utilisées pour les trois premières dans l'enseignement secondaire - deux Bescherelle (Guillon 1990 [B90] ; Bescherelle 1997 [B97]) et un Bled Grammaire (Berlion 2015 [B15]) -, et pour les deux dernières dans l'enseignement supérieur - la Grammaire méthodique du français (Riegel, Pellat et Rioul 2011 [GMF]) et la Grammaire du français (Denis et Sancier-Château 1994 [GF]). Les passages concernant les piliers du masculin apparaissent en gras pour l'accord 
au masculin, soulignés pour le masculin générique et en italiques et soulignés pour le masculin forme première :

B90: «l'adjectif épithète s'accorde en genre et en nombre avec le
nom qu'il qualifie. [...] Lorsque l'adjectif qualifie plusieurs noms
de genres différents, il se met au masculin pluriel » (p. 9); «En
principe, le e final est la marque du féminin » (p. 193).

B97 : « Le genre est marqué par : un changement de la fin du nom (un chat, une chatte), un changement de forme (un cerf, une biche), aucun changement (un collègue, une collègue)" (\$253); "La plupart des noms de profession changent au féminin (un boulanger $\rightarrow$ une boulangère) $)(\S 256)$.

B15 : « On forme généralement le féminin en ajoutant un -e au nom masculin $»($ p. 14)

GF : «Un certain nombre de noms peuvent s’appliquer à des femmes tout en restant au masculin » (p. 350).

GMF : «Si les noms sont de genre différent, l'adjectif se met généralement au pluriel et au masculin (qui est la forme non marquée du point de vue du genre) » (p. 611) ; «L'opposition est généralement neutralisée au profit du nom masculin lorsqu'il entend désigner l'espèce entière sans distinction de sexe » (p. 331).

Dans les cinq ouvrages considérés, au moins un des trois piliers est évoqué (B15, B97), parfois deux (GF, B90) ou les trois (GMF). Quoi qu'il en soit, malgré la reconnaissance d'exceptions ( « généralement », GMF, B15 ; «parfois », GF ; « la plupart », B97 ; « En principe », B90), ce qui fonde et justifie les phénomènes évoqués, ce qui permet d'en comprendre l'émergence et la stabilité n'est en aucun cas questionné.

De plus, ces phénomènes sont presque toujours associés à une distinction première, présente cette fois dans quatre des cinq ouvrages (le B90 n’étant pas centré sur le lexique), et qui concerne plus particulièrement les noms d'humains (NH) :

B97 : « Pour le nom des êtres animés, le genre dépend du sexe de l’être désigné » (§ 253).

B15: «[L]es noms d’êtres animés sont ordinairement du masculin ou du féminin suivant le sexe » (p. 14).

GF : «Pour les noms référant à des animés (humains ou non), l'opposition des sexes conduit parfois à une opposition en genre » (p. 349-350).

GMF : «Les noms animés constituent une sous-classe où la distinction des genres correspond en règle générale à une distinction de sexe » (p. 329).

En parcourant ces ouvrages descriptifs, on apprend donc d'emblée la binarité du genre grammatical (féminin, masculin, et rien d'autre), ainsi que le lien entre cette binarité linguistique et une binarité non-linguistique : le genre grammatical, dans le cas des $\mathrm{NH}$, est présenté comme un indicateur transparent de bascule de la sous-catégorie référentielle /mâle/ à la sous-catégorie référentielle /femelle/, ou inversement. Selon cette conception, 
l'opposition entre les deux manifestations du genre grammatical représenterait en langue ce que les autrices de la GF nomment explicitement « l'opposition des sexes » : mâle, femelle, et rien d'autre. Mais à cette double binarité (référentielle et grammaticale) vient s’ajouter l'idée que l' «opposition » en question n'est pas symétrique et que l'un des deux genres grammaticaux tient une position secondaire par rapport à l'autre : le féminin est dérivé du masculin, n’accède pas à la «valeur générique » et cède le pas au masculin dans les cas d'accords complexes. Ce déséquilibre n’est pas explicitement posé comme lié à un déséquilibre extra-linguistique, mais d’emblée, la corrélation entre genre grammatical et sexe laisse la brèche ouverte, d'autant plus du fait de l'absence de toute problématisation de cette notion de « sexe ».

L’idée ainsi posée d'une primauté du genre grammatical masculin, bien qu'extrêmement problématique, n'est pas propre aux ouvrages descriptifs, mais trouve un de ses appuis dans les théories de la marque développées entre autres à partir des travaux de R. Jakobson et $\mathrm{N}$. Troubetzkoï, et qui du phonologique se sont étendues au morphologique et, à terme, au sémantique : à une forme non-marquée morphologiquement et sémantiquement (le masculin : forme première, avec un sens potentiellement générique), s’oppose une forme marquée morphologiquement et sémantiquement (le féminin : forme seconde, avec un sens toujours spécifique). Le féminin est, dans les faits, " posé comme inexistant au départ » (Khaznadar 2002, p. 152).

\title{
1.2 Pratiques lexicographiques
}

Cette idée d’une secondarité du genre grammatical féminin est quasi-omniprésente dans les ouvrages grammaticaux contemporains, bien que plus ou moins explicitement posée. C'est à Marguerite Durand, en 1936, que l'on doit une des descriptions les plus claires de l'asymétrie entre les genres grammaticaux:

\begin{abstract}
" [L]es formes masculines et féminines ne sont pas, dans notre esprit, des symétriques exactes; nous nous rappelons, nous pensons le mot sous sa forme masculine; celle-ci ne se présente pas à notre esprit comme un mot pourvu d'un genre ou d'une forme quelconque, c'est le mot lui-même ; le féminin n'en est que la forme dérivée, de même que dans le français écrit, il est aussi dans la langue parlée une forme plus compliquée et secondaire » (Durand 1936, p. 27)
\end{abstract}

Ce qui est intéressant dans l'analyse de M. Durand, c'est qu'elle ne s'attarde en rien sur la dimension sémantique de cette répartition, mais bien plutôt sur son aspect formel et cognitif : le mot «ne se présente pas à notre esprit » sous sa forme féminine, mais est enregistré comme masculin. Comme le souligne Claire Michard (Michard 2002, p. 62-63), la psychologisation est en fait ici équivalente à un processus de naturalisation, qui fait de la primauté du masculin un des critères intrinsèques, systémiques (et non pas historiques et/ou sociaux) de la catégorie du genre grammatical en langue française.

La notion de naturalisation peut être éclairée par l'analyse en termes de condition et de position, développée entre autres par Maria Puig de la Bellacasa (2003) : la condition renvoie à l'essentialisation de traits caractéristiques considérés comme inhérents et/ou innés (les diverses conditions seraient au fondement des différenciations sociales) ; la position est au contraire politique et existentielle (elle découle des rapports de domination, peut parfois les renforcer, mais ne les cause pas). La délimitation des groupes sociaux selon la position (et non plus la condition) permet de problématiser les rôles et fonctions qui leur sont usuellement associés. Elle invite aussi à revenir sur la nécessité même de penser une 
dichotomie première entre mâles et femelles : cette dichotomie serait justement l'effet de la naturalisation de la position sociale, alors perçue comme condition. Il ne s'agit évidemment pas, avec l'introduction de cette notion, de nier l'existence des organes génitaux et de leurs traductions physiologiques ${ }^{5}$, mais plutôt de dénoncer l'idée d'une naturalité spécifiquement féminine (ou masculine). La critique des processus naturalisants permet donc de montrer que la « nature » qui fonde la bicatégorisation n'est pas signifiante a priori : elle est « ellemême naturalisée » (Puig de la Bellacasa 2003, p. 41).

La naturalisation par «l'esprit », lisible dans les propos de M. Durand, se consolide dans les productions grammaticales évoquées ci-dessus, mais trouve aussi un de ses points d'ancrage et de pérennisation principaux dans les pratiques lexicographiques du genre grammatical, qui posent systématiquement la forme masculine des mots dits « variables en genre » comme lemmatique, quelle que soit l'histoire réelle du mot. Dans nos recherches, nous retenons les six ouvrages suivants: le Trésor de la langue française informatisé (ATILF) ; les huitième et neuvième éditions du Dictionnaire de l'Académie française (AF8, AF9) ; l'édition de 2015 du Petit Robert (PR15) ; le Dictionnaire langue française en ligne de Larousse (LAR) ; le Nouveau Littré (NLIT).

Tableau 1. Le masculin lemmatique.

\begin{tabular}{|c|c|c|c|c|c|}
\hline & $\begin{array}{l}\text { Forme } \\
\text { masc. + } \\
\text { forme } \\
\text { fém. }\end{array}$ & $\begin{array}{l}\text { Forme masc. + } \\
\text { morphèmes fém. }\end{array}$ & $\begin{array}{l}\text { Forme masc. + } \\
\text { fém. mentionné } \\
\text { dans l'article }\end{array}$ & $\begin{array}{l}\text { Forme masc. } \\
\text { seule }\end{array}$ & $\begin{array}{l}\text { Forme fém. } \\
\text { seule }\end{array}$ \\
\hline Avocat, avocate & LAR & $\begin{array}{l}\text { ATILF ; AF9 ; } \\
\text { PR15 ; NLIT }\end{array}$ & AF8 & $\varnothing$ & $\varnothing$ \\
\hline $\begin{array}{l}\text { Boucher, } \\
\text { bouchère }\end{array}$ & LAR & $\begin{array}{l}\text { ATILF ; AF9 ; } \\
\text { PR15 }\end{array}$ & AF8 & NLIT & NLIT \\
\hline $\begin{array}{l}\text { Cavalier, } \\
\text { cavalière }\end{array}$ & LAR & $\begin{array}{l}\text { ATILF ; AF8 ; } \\
\text { AF9 ; PR15 ; } \\
\text { NLIT }\end{array}$ & $\varnothing$ & $\varnothing$ & $\varnothing$ \\
\hline $\begin{array}{l}\text { Couturier, } \\
\text { couturière }\end{array}$ & LAR & ATILF & AF8 & $\begin{array}{l}\text { AF9 ; PR15 ; } \\
\text { NLIT }\end{array}$ & $\begin{array}{l}\text { AF9 ; PR15 ; } \\
\text { NLIT }\end{array}$ \\
\hline $\begin{array}{l}\text { Cuisinier, } \\
\text { cuisinière }\end{array}$ & LAR & $\begin{array}{l}\text { ATILF ; AF8 ; } \\
\text { AF9 ; PR15 ; } \\
\text { NLIT }\end{array}$ & $\varnothing$ & $\varnothing$ & $\varnothing$ \\
\hline $\begin{array}{l}\text { Financier, } \\
\text { financière }\end{array}$ & $\varnothing$ & $\begin{array}{l}\text { ATILF ; PR15 ; } \\
\text { NLIT }\end{array}$ & $\varnothing$ & $\begin{array}{l}\text { AF8 ; AF9 ; } \\
\text { LAR }\end{array}$ & $\varnothing$ \\
\hline $\begin{array}{l}\text { Intendant, } \\
\text { intendante }\end{array}$ & LAR & $\begin{array}{l}\text { AF8 ; AF9 ; } \\
\text { PR15 ; NLIT }\end{array}$ & $\varnothing$ & ATILF & ATILF \\
\hline Préfet, préfète & $\varnothing$ & $\varnothing$ & AF8 & $\begin{array}{l}\text { ATILF ; AF9 ; } \\
\text { PR15 ; LAR ; } \\
\text { NLIT }\end{array}$ & $\begin{array}{l}\text { ATILF ; AF9 ; } \\
\text { PR15 ; LAR }\end{array}$ \\
\hline $\begin{array}{l}\text { Professionnel, } \\
\text { professionnelle }\end{array}$ & LAR & $\begin{array}{l}\text { ATILF ; AF8 ; } \\
\text { AF9 ; PR15 ; } \\
\text { NLIT }\end{array}$ & $\varnothing$ & $\varnothing$ & $\varnothing$ \\
\hline
\end{tabular}

En parcourant ces quelques ouvrages lexicographiques, on constate assez rapidement qu'à chaque fois qu'une entrée fait apparaître la variation en genre, la forme masculine 
arrive la première (Forme masc. + forme fém. ; Forme masc. + morphèmes fém.). De plus, un seul des six ouvrages utilisés propose les deux formes complètes (LAR) : les autres dictionnaires présentent la forme masculine complète, puis les morphèmes de genre grammatical féminin (-ate, -ère, -ière, -ante, -elle). ${ }^{6}$ Ces pratiques lexicographiques n’ont pas de justification scientifique particulière: l'apparition du masculin en premier peut s'expliquer par l'organisation alphabétique adoptée par les dictionnaires, mais la présentation d'une forme masculine complète et d'une forme féminine tronquée (ne reste que la marque) ne s'explique pas par des critères linguistiques ${ }^{7}$, surtout quand le substantif en question est issu de dérivation. Ces pratiques, solidement instituées, tout comme celles des ouvrages de grammaire cités plus haut, secondarisent la forme féminine de ces dénominations de la personne en la posant comme simplement affixale. La primauté du masculin est présentée comme systémique: dans le cas des noms variables, comme l'écrivait M. Durand, il apparaît bien, avec les outils dont nous disposons, que le masculin, « c’est le mot lui-même ».

\subsection{La « valeur générique » du masculin}

L'explicitation d'un déséquilibre entre les deux manifestations du genre grammatical n'est toutefois pas propre aux productions scolaires et lexicographiques. Les questionnements autour de cette catégorie se retrouvent dans de nombreux types de discours différents, allant des déclarations institutionnelles aux échanges informels. Et en effet, c'est le troisième pilier de la primauté du masculin (la «valeur générique »), qui, plutôt discret dans les ouvrages susmentionnés, est prédominant dans les discours non-spécialisés. Cette spécificité du genre grammatical masculin ne peut exister que dans le cadre de la dénomination de la personne, et ne concerne (a priori) que les noms variables en genre : le masculin serait apte à dénommer indifféremment au sexe des référents visés, et constituerait un palliatif à la binarité masculin/féminin, et peut-être même à la binarité mâle/femelle. C'est en tout cas de cette façon que le présente l'Académie Française, dans son billet d’octobre 2014 sur la question de la féminisation des noms de métiers :

«L'une des contraintes propres à la langue française est qu'elle n’a que deux genres: pour désigner les qualités communes aux deux sexes, il a donc fallu qu'à l'un des deux genres soit conférée une valeur générique afin qu'il puisse neutraliser la différence entre les sexes. L’héritage latin a opté pour le masculin » (Académie 2014).

La «valeur générique » permet bien, si l’on en croit ce propos, de dépasser l’idée du genre grammatical comme indicateur de bascule référentielle, mais uniquement pour « l'un des deux genres » : le féminin indique nécessairement la restriction à la sous-catégorie référentielle /femelle/, alors que le masculin peut s'extraire de cette dichotomie. Ce qui est particulièrement intéressant ici, c’est l'équivalence posée entre cette valeur propre au masculin et le genre grammatical «neutre». Mais cette idée repose en fait sur une confusion terminologique, masquée par la référence au très autoritaire " héritage latin » : le neutre latin est un genre grammatical à part entière, qui se caractérise par une différenciation morphologique par rapport au féminin et au masculin, et non par une capacité à « neutraliser la différence entre les sexes », donc à référer à des entités /mâles/ et/ou /femelles/. En ce sens, la langue française n'est pas en manque par rapport à la langue latine. Selon la conception de l'Académie, le «neutre ", de genre grammatical à part entière, devient un surplus sémantique qui colore un genre grammatical déjà existant ${ }^{8}$. Cette « valeur », qui introduit en outre une confusion avec les « emplois génériques »" ${ }^{9}$, qui 
eux concernent tous les substantifs, est posée par l'Académie comme indispensable à la cohérence de la langue, et à terme, indiscutable. C'est la linguiste Claire Michard qui propose la lecture la plus riche et la plus convaincante de cette problématique :

\begin{abstract}
«[C]'est bien parce que le sens premier du masculin ne pose rien quant au sexe qu'il est capable de référer à des humains hors mention de sexe, ou avec mention de sexe mâle, ou avec mention de sexe femelle. Et c'est bien parce que le signifié de sexe est premier pour le féminin qu'il est incapable de référer à des humains hors mention de sexe » (Michard 1999, p. 79-80)
\end{abstract}

Au genre grammatical masculin correspondraient les traits [+ humain] et/ou [+ mâle], et au genre grammatical féminin, le seul trait [+ femelle]. La forme couturier serait donc apte à désigner la sous-catégorie /couturier/ ou la catégorie /couturier, couturière/, alors que la forme couturière serait limitée à un renvoi à la sous-catégorie /couturière/. La "valeur générique » constituerait donc une potentialité hyperonymique (Chevalier 2013) propre à la seule forme masculine des dénominations de la personne variables en genre. La secondarisation du genre grammatical féminin viendrait donc du fait qu'il restreint au renvoi catégoriel en fonction du sexe, donc en fonction d'un critère discriminant. En ce sens, le fonctionnement de la "valeur générique » (mis en avant par l'Académie française comme «neutralisant ») s'apparenterait plutôt à celui d'un genre "commun » (l'un et l'autre) que « neutre » (ni l'un ni l'autre) ${ }^{10}$.

C'est sur cet argument du renvoi hyperonymique propre au masculin que se fondent souvent les discours de rejet des processus de féminisation des noms de métiers ${ }^{11}$, malgré l'isomorphisme entre masculin référant au sexe et masculin à valeur générique :

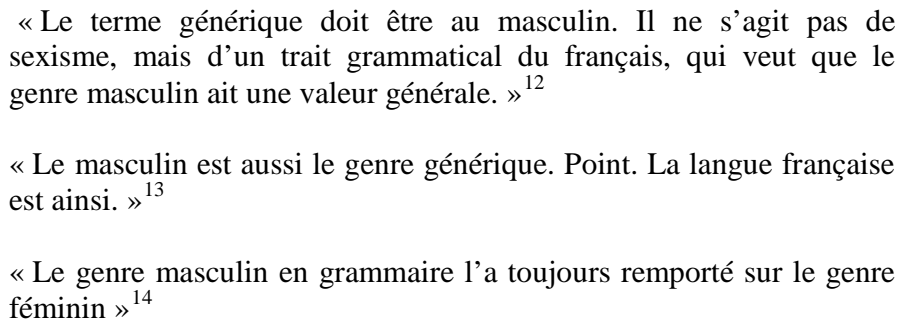

À la lecture de ces extraits, on peut rapidement constater deux choses : la croyance en la prédominance intrinsèque du genre grammatical masculin est liée à un refus du changement linguistique, donc à une forme de fixisme («La langue française est ainsi »; " Le genre masculin [...] l'a toujours remporté ») ; ce fixisme s'appuie sur la stratégie rhétorique de l'argument d'autorité (présent gnomique, adverbe temporel toujours, modalités déontique et/ou aléthique, etc.). Le point commun entre ces deux éléments est l'effort assez évident de déshistoricisation et de décontextualisation de la langue, qui est posée comme dénuée de tout lien avec le monde extra-linguistique, et plus simplement, avec la société ( « il ne s’agit pas de sexisme, mais d'un trait grammatical du français »). Et c'est de ce même effort que participent, à leur manière, les grammaires et dictionnaires évoqués ci-dessus : la primauté du masculin est pensée comme une vérité linguistique, sans histoire, sans contexte, donc comme une loi grammaticale n'ayant nullement besoin de justification.

L'approche constructiviste du genre grammatical s'attache au contraire à le sortir des effets apriorisants des descriptions usuelles. Ainsi, nous inscrivons notre propos dans la lignée du féminisme matérialiste théorisé par Christine Delphy (1977), Nicole-Claude Mathieu (1991) ou encore Colette Guillaumin (1992), dont l'enjeu principal est la 
visibilisation des processus dont émerge la hiérarchie entre les genres (sociaux), et par ricochet, entre les sexes. Cet enjeu, du fait du lien souvent réaffirmé entre sexe et genre grammatical, est aisément transposable aux dénominations de la personne. Et en effet, l'approche linguistique matérialiste s'applique à reconnecter la langue au monde social et culturel dans lequel elle est pratiquée, en prenant en compte les rapports de force et de domination qui le structurent et rendent ses objets signifiants (Michard 2003, p. 77-78). La réflexion sur la naturalisation évoquée ci-dessus permet de concilier cette approche matérialiste avec les théories queer, développées notamment par Judith Butler (1995) et centrées sur la théorie psycho-discursive de la performation des genres ${ }^{15}$. En effet, comme le rappelle la philosophe Elsa Dorlin :

\begin{abstract}
«Ce n'est pas parce que tout est construit - notamment au travers du discours - que ce n'est pas réel, que la domination n'est pas matérielle. Ainsi, ce n'est pas parce que les identités «homme " ou "femme » sont construites qu'elle ne sont pas réelles, oppressantes et oppressives » (Dorlin 2007, p. 54)
\end{abstract}

La perspective diachronique que nous proposons vise à mettre au jour les mécanismes qui sous-tendent, stabilisent et banalisent la primauté du genre grammatical masculin, et s'inscrit donc parfaitement dans cette démarche. En effet, s'attacher à dévoiler la construction du rapport entre sexe et genre grammatical ne revient pas à nier l'existence ce rapport, mais permet au contraire d'en comprendre les fondements matériels (théoriques et pratiques).

\title{
2 L'établissement du masculin premier : penser le genre en diachronie
}

\subsection{XVlème-XVIlème siècles : sexe, genre, accord - théories et pratiques de linguistes}

Afin de comprendre ces processus, nous évoquerons un ensemble d'ouvrages des XVIème et XVIIème siècles, pour la raison historique et politique que, comme le rappelle Sylvain Auroux, « on date ordinairement du XVIème siècle la prise de conscience de l'autonomie et du prestige de la langue romane nommée «français » » (Auroux 1992, p. 359) ${ }^{16}$. À la lecture de ces ouvrages, on constate avant tout l'omniprésence, à une période où les questionnements sur l'origine du langage et des langues sont prégnants, de l'hypothèse d'un rapport motivé entre sexe et genre grammatical (dans cet ordre là). Cette motivation, qui se retrouve dans les ouvrages descriptifs évoqués plus haut, est explicitée dès les toutes premières publications grammaticales sur la langue française :

\footnotetext{
« Come natur' eyt a bone rezon inuenté le mal' e la fumelle pour sa conservaçion, e continuaçion de ses especes, l'artific'aosi du langaje a (en la suyuant come gyde) doné ao noms note de masculin, e femenin » (Meigret 1550, p. 34)
}

La langue traduirait donc par le genre grammatical une dichotomie première, entre mâles et femelles. C'est cette idée qui va parcourir l'ensemble des ouvrages du siècle et de celui qui suit : 
« Le genre est masculin ou feminin. Si le nom conuient au masle, il est masculin : sil conuient à la femelle, il est femenin » (Pierre de la Ramée 1572, p. 60-61)

«Premierement les noms propres de Dieux, d'hommes, d'Anges, \& de Démons sont masculins [...]. Tous noms de dignitez \& d'offices appartenans à l'homme sont masculins [...]. Mais les noms propres de femmes sont feminins [...]. D’offices \& conditions appartenantes aux femmes » (Oudin 1632, p. 51-52)

On note que, déjà chez Antoine Oudin, les enjeux socio-culturels sont présents dans l'explicitation de la motivation entre sexe et genre. La répartition en genre des substantifs serait alors liée à la bipartition sexuée, mais aussi aux activités considérées comme propres à «l'homme » (et non plus au " masle ») ou aux «femmes » (et non plus à la " femelle »), donc à des pratiques socialement et culturellement différenciées. Toutefois, en analysant ces extraits, il ne faut pas tomber dans un travers anachronisant prêtant à l'auteur une volonté de différencier entre un sexe qui serait " naturel » et des pratiques " culturelles » (déterminant ce qu'on appellerait aujourd'hui le genre ?). En effet, il est important de tenir compte du fait que, dans la pensée dominante du XVIème siècle, les rôles sociaux et culturels sont tout aussi naturels que la distinction première entre les sexes. Ainsi, comme le précise l'historienne S. Steinberg :

« Différence des sexes et différence sociale sont toutes deux pensées dans le cadre d'une même philosophie de la nature, inspirée de celle d'Aristote, et procèdent d'une même vision cosmologique qui relie “ce petit monde” qu'est l'homme aux éléments du Cosmos auquel il appartient. » (Steinberg 2010, p. 138)

Les deux niveaux ne sont pas dissociés, et les rapports de force qui s'instaurent au sein de la société découlent de la nature de ses composantes, et des accidents de leur rencontre. Ainsi, malgré l'élargissement, dans la présentation du genre grammatical, à des critères qui seraient aujourd'hui pensés comme non-naturels, les distinctions qu'ils visibilisent sont généalogiquement ( «premierement ») fondées sur la répartition entre les sexes.

Cette motivation première, qui soutient la nécessité d’une répartition genrée des substantifs, trouve sa justification la plus explicite dans la Grammaire générale et raisonnée d'Arnauld et Lancelot :

«Or les hommes se sont premierement considerez eux-mesmes, \&
ayant remarqué parmy eux une difference extrémement considerable,
qui est celle des deux sexes, ils ont jugé à propos de varier les mesmes
noms adjectifs, y donnant diverses terminaisons, lors qu'ils
s'appliquoient aux hommes, \& lors qu'ils s'appliquoient aux
femmes » (Arnauld et Lancelot 1660, p. 39)

Dans cette analyse des deux grammairiens, l'argument qui sous-tend l'émergence des genres grammaticaux est non pas linguistique, mais bien fondé sur le rapport à la nature, et historiquement déterminé, bien que cette détermination demeure hypothétique : c'est en se « considérant eux-mêmes » que les êtres humains auraient décidé de la validité d'une catégorie grammaticale. Ici, le non-linguistique est pris en compte comme éclairant le linguistique ${ }^{17}$. C'est à partir de ce point de départ que l'on peut saisir certaines évolutions de la compréhension de cette catégorie grammaticale. Un des exemples les plus frappants est dû à Charles Maupas (1607), qui affirme dans sa Grammaire et syntaxe françoise que : 
"Plusieurs substantifs masculins en font sortir d'eux, d'autres feminins suyvant la formation des Adjectifs susdits » (Maupas 1607, p.81)

L’idée du masculin comme forme première du nom, comme forme non-altérée, et plus tard, avec la formalisation des dictionnaires, lemmatique, déborde du simple domaine morphologique, du fait de ce lien serré entre genre grammatical et sexe. Le fondement culturel et naturalisant donné au genre grammatical se retrouve dans cette idée de la primauté (ici pensée comme génétique) du masculin : ce féminin «sorti » du masculin, dans la société française des XVIème et XVIIème siècles, a très certainement pour toile de fond le récit chrétien des origines, selon lequel Eve est sortie d'Adam. Et en effet, on ne peut dissocier l'assertion généralisée selon laquelle «le feminin est formé du masculin » (Ramée 1572, p.64) de la pensée sous-jacente, fondée sur l’idée d’une saillance naturelle du sexe féminin comme critère de différenciation. C'est aussi sous ce jour que l'on peut lire l'émergence, au XVIIème siècle, de «l'accord au masculin ». L'ouvrage qui, concernant cette question, sert parfois encore de référence, est celui de Claude Favre de Vaugelas, publié en 1647. En effet, l'auteur, dans ses Remarques sur la langue françoise, propose l'analyse suivante, fondée sur une phrase de Malherbe («Ce peuple a le cœur et la bouche ouverte à vos louanges ») :
« Il faudrait dire ouverts selon la Grammaire Latine, qui en use ainsi, pour une raison qui semble être commune à toutes les Langues, que le genre masculin étant le plus noble, doit prédominer toutes les fois que le masculin \& le féminin se trouvent ensemble » (Vaugelas 1647, p. 264)

C'est cette assertion qui a largement été retenue (parfois reprise telle quelle, parfois modifiée). Mais à celle-ci, Vaugelas ajoute immédiatement :

\begin{abstract}
« Je voudrais dire ouverte, qui est beaucoup plus doux, tant à cause que cet adjectif se trouve joint au même genre avec le substantif qui le touche, que parce qu'ordinairement on parle ainsi, qui est la raison décisive, \& que par conséquent l'oreille y est toute accoutumée » (idem, p. 264)
\end{abstract}

L'auteur oppose donc immédiatement à la règle de raison, fondée sur la noblesse d'un genre grammatical par rapport à l'autre, la pratique effective de son temps ${ }^{18}$, qui tend à privilégier l'accord de proximité, aujourd'hui largement oublié. C'est en fait à la fin du XVIIIème siècle que Nicolas Beauzée explicite et radicalise le propos de Vaugelas, en restituant la règle mais non la discussion :

" Si un adjectif se rapporte à plusieurs noms appellatifs de différents genres, il se met encore au pluriel, et il s'accorde en genre avec celui des noms qui est du genre le plus noble » (Beauzée 1767, p. 627)

Ce à quoi l'auteur ajoute immédiatement, reprenant l'idée de noblesse présente mais non développée chez Vaugelas (et sur laquelle il était encore possible de s’interroger) :

« Le genre masculin est réputé plus noble que le féminin à cause de la supériorité du mâle sur la femelle » (idem, p. 627)

Un siècle après Vaugelas, la noblesse du genre grammatical masculin est très explicitement corrélée à une certaine conception de la domination masculine, présentée comme essentielle, naturelle et indiscutable : la primauté du mâle justifie la primauté du masculin. L'accord de proximité, intra-linguistique, cède le pas à un accord par défaut au masculin, fondé sur l'idée non-linguistique que la matrice de l'humain est le mâle. On trouve donc ici, dans l'histoire même des représentations liées au genre grammatical, le 
fondement de la théorie proposée plus de deux siècles plus tard par Claire Michard (1996, 1999, 2002) . Sur cette supériorité, la «valeur générique du masculin » peut aisément se développer et se stabiliser, et la distance n'est pas grande entre un genre grammatical masculin qui peut reprendre le masculin et le féminin et un genre grammatical masculin qui peut référer indifféremment aux individus /mâles/ et/ou /femelles/.

\subsection{XVIIIème-XIXème : construction de l'argument d'autorité}

La période suivante favorise largement la transmission de ces conceptions, entre autres du fait des évolutions culturelles et politiques enclenchées dès la fin du XVIIIème siècle ${ }^{19}$. Durant cette période de massification des productions linguistiques et surtout de leur diffusion, on note, concernant la question du genre grammatical, deux tendances. La première reprend et valide largement la conception naturalisante du genre grammatical développée durant les siècles précédents. Dans tous les ouvrages étudiés, on la retrouve explicitement, généralement en tête des articles sur le genre grammatical :

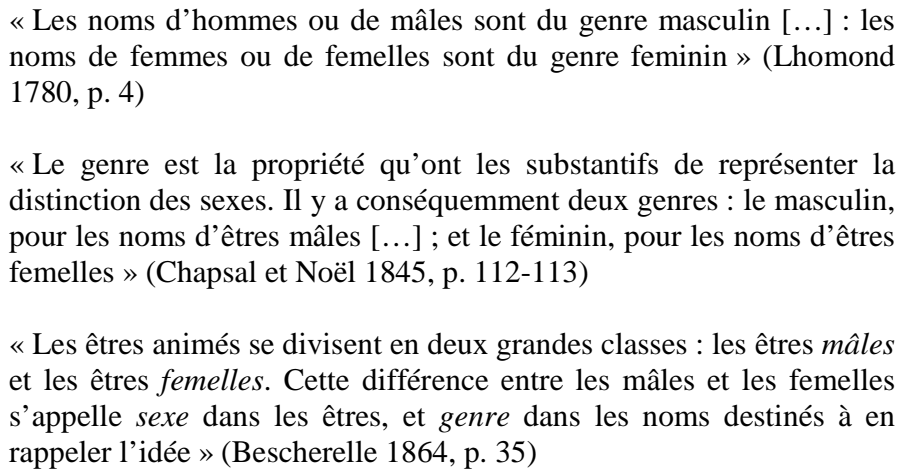

" Les êtres animés se divisent en deux grandes classes : les êtres mâles et les êtres femelles. Cette différence entre les mâles et les femelles s'appelle sexe dans les êtres, et genre dans les noms destinés à en rappeler l’idée » (Bescherelle 1864, p. 35)

Le genre grammatical, comme aux XVIème et XVIIème siècles, est posé comme nécessairement lié au sexe. Mais l'explication téléologique que proposaient à la fois Meigret (1550) et les auteurs de la Grammaire générale (Arnauld et Lancelot 1660) disparaît au profit d'une assertion gnomique. La naturalisation, en s'effaçant comme processus et en ne laissant apparaître que le résultat final, est donc amenée à son terme ${ }^{20}$ : elle diffère de la tendance des siècles précédents en ce qu'elle n’est plus explicitement fondée sur une compréhension naturalisante de la question, mais sur une dichotomie donnée comme un fait indubitable, et premier.

Face à cela, on trouve une tendance, en apparence opposée, à la dénaturalisation des problèmes liés à la catégorie du genre grammatical. Celle-ci est particulièrement explicite dans le cas de l' « accord au masculin » :

«Quand un Adjectif se rapporte à deux noms singuliers, on met cet adjectif au pluriel [...]. Si les deux sont de différents genres, on met l'adjectif au masculin. » (Lhomond 1780, p. 11)

«377. S'il y a deux ou plusieurs substantifs ou pronoms, l'adjectif se met au pluriel, et prend le genre masculin, si les substantifs ou les pronoms sont de différents genres. » (Chapsal et Noël, 1845, p. 118)

«365. Lorsque les noms sont de différent genre, l'adjective qui s’y rapporte se met au masculin » (Bescherelle 1841, p. 117) 
Dans ces extraits, aucune mention de la noblesse du masculin, ni de la supériorité du mâle sur la femelle. La justification masculiniste, l'explication sociale - pensée comme découlant de la nature des individus - qui était originellement attachée à la préférence donnée à l'accord au masculin sur l'accord de proximité disparaît complètement. L'effacement du lien entre le phénomène non-linguistique et le phénomène linguistique fait qu'à la fin, il ne reste que la loi, l'argument d'autorité qui pose l'incontestabilité du phénomène linguistique.

On trouve donc dans les ouvrages de la fin du XVIIIème et du XIXème siècle un double mouvement d'assertion du lien motivé entre sexe et genre grammatical, et d'abstraction de règles détachées de ce lien premier. Ce phénomène d’abstraction permet et renforce l’idée d’une hiérarchie, non plus fondée sur une idéologie, mais structurellement nécessaire, entre les deux manifestations du genre grammatical. Ces tendances en apparence contradictoires peuvent en fait être pensées comme fermement liées. En effet, le rapport naturel entre genre et sexe, à partir de la fin du XVIIIème siècle, n'est plus posé comme explicatif, mais comme descriptif, et de la même façon, les effets qui en découlent sont pensés comme des axiomes : la forme masculine est première, l'accord par défaut se fait au masculin, seul le masculin peut porter la "valeur générique ». L’invisibilisation progressive des processus qui mènent à ces lois assoit la primauté d'un genre grammatical sur l'autre, en la posant comme inhérente au système linguistique français.

\section{Repenser le rapport entre masculin et féminin : sémantique du genre grammatical}

\subsection{L'effort d'historicisation : vers l'hypothèse du « culturel »}

L'historicisation de la catégorie permet donc déjà en partie d'expliciter l'idéologie qui sous-tend le rapport entre les deux genres grammaticaux. Une des façons d'illustrer ce point consiste dans l'étude de l'évolution des traitements lexicographiques des dénominations de la personne. Nous en retiendrons ici un exemple, le binôme avocat, avocate.

Les formes avocat et avocate sont citées dans l'ouvrage d'Antoine Oudin, au sein d'une liste de substantifs de dénomination de la personne variables en genre, sans distinction sémantique particulière :

« Premièrement les noms d'offices \& métiers terminés en er [...].

Apres cela des noms terminez en $d \& t$ Marchand, Marchande: Aduocat, Aduocate » (Oudin 1632, p. 11-12).

Mais dès les premiers grands ouvrages lexicographiques du XVIIème siècle, une différenciation est introduite, et se manifeste à la fois dans le contenu des entrées et dans leur présentation :

«Avocat. s. m. Celui qui en vertu de ses licences \& de sa matricule plaide \& défend en justice les gens qui ont besoin de lui. [...] Celui qui soutient \& défend les intérêts de quelque personne »; "Avocate. s.f. [...] Celle qui prend nos intérêts, quelques uns croient qu'il faut dire en ce sens, avocat, \& non avocate. [...] Cependant il semble que l'usage veuille que dans cette phrase on dise avocate » (Richelet 1680). 
«ADVOCAT, s.m. Homme sçavant en Jurisprudence, qui deffend de vive voix, ou par écrit le droit des parties qui ont besoin de son assistance. [...] Advocat, ate, se dit figurément de celuy ou de celle qui prend à coeur les interests, ou les affaires de quelqu'un [...] » (Furetière 1690).

"ADVOCAT. s.m. Celuy qui fait profession de deffendre des causes en Justice. [...] Aduocat, se dit fig. de Celuy qui intercede pour un autre [...]. En ce sens on dit aussi Advocate. » (Académie 1694)

Dans les trois dictionnaires, le choix de la forme masculine en vedette est justifié référentiellement : "Celui qui » (Richelet 1680, Académie 1694), "Homme sçavant » (Furetière 1690) renvoient explicitement à des référents /mâles/. La seule trace de ce qui pourrait correspondre à une "valeur générique » du masculin apparaît dans la remarque proposée par Richelet : «quelques uns croient qu'il faut dire en ce sens, avocat, \& non avocate ». Mais d'emblée, cette idée est nuancée par le renvoi à l' " usage ", qui, tout comme le précisait Vaugelas concernant l'accord au masculin, contredit au moins en partie cette « croyance ». La mise en vedette de la forme masculine paraît donc directement liée à une question de représentativité : dans la perspective largement naturalisante du XVIIème siècle, l'actualisation privilégiée des morphèmes grammaticaux de genre masculin se justifie pleinement par le fait que le référent majoritaire (et même obligatoire) de la catégorie professionnelle appartient à la sous-catégorie /mâle/. Ainsi, la forme avocate persiste, mais n'est présentée en vedette que dans le Richelet, qui traite les formes masculine et féminine séparément.

On retrouve la même tendance dans les dictionnaires des XVIIIème et XIXème siècles, avec toutefois un glissement progressif vers l'acceptation de la "valeur générique » du masculin :

"AVOCAT, s.m. celui qui fait profession de défendre des causes en Justice. [...] Se dit figur. De celui qui intercède pour un autre, qui en soutient, qui en défend les intérêts auprès de quelqu'un. [...] En ce sens, on dit aussi, Avocate » (Académie 1740)

"AVOCAT, ATE, s.m. et f. Au propre, on ne le dit guère qu'au masculin, de celui qui fait profession de défendre des causes en Justice - [...] l'Acad. ne met Avocate qu'au figuré et en parlant la Ste. Vierge » (Féraud 1787)

"AVOCAT, sub. Mas. Celui qui fait profession de défendre des causes en Justice. [...] Se dit figurément de celui qui intercède pour un autre, qui en soutient, qui en défend les intérêts auprès de quelqu'un. [...] En ce sens, on dit aussi Avocate. Sa mère fut son avocate" (Académie 1799)

" AVOCAT, s.m. Celui qui fait profession de défendre en justice, de vive voix ou par écrit, ceux qui ont besoin de son assistance [...]. En parlant d'une femme qui exercerait la profession d'avocat, on dirait, Un avocat. Melle d'Eon fut mise à 14 ans au college Mazarin ; on ignore les raisons qui engagerent ses parents à lui donner l'habit d'homme. Elle fut reçue docteur en droit civil et en droit canon, et enfin avocat au parlement de Paris. [...] Fig. Celui qui intercède pour un autre, qui en soutient, qui en défend les intérêts auprès de 
quelqu'un [...]. En ce sens, on dit avocate. Sa mère fut son avocate » (Bescherelle 1856)

«AVOCAT, n.m. Celui qui fait profession de défendre en justice. Fig.

Celui qui intercède pour un autre. Dans ce sens il y a un feminin :

l'avocate des pécheurs, la sainte Vierge » (Larousse 1886)

Dans son sens figuré, le mot avocat.e conserve ses deux formes, sans que cela semble poser de difficultés. Cependant, la remarque de Bescherelle sur les femmes exerçant la profession marque un tournant intéressant dans la réflexion sur le genre grammatical proposée par les lexicographes. L’énoncé «En parlant d’une femme qui exercerait la profession d'avocat, on dirait, Un avocat» pourrait laisser penser que la "valeur générique » attribuée au masculin est pleinement établie dans le domaine non-métaphorique des noms de fonctions, mais la précision qui suit pose le problème un peu différemment : « on ignore les raisons qui engagerent ses parents à lui donner l'habit d'homme ». Le seul exemple d'une femme de la profession concerne en fait un cas de mimétisme social : se faire passer pour un homme, pour exercer un métier usuellement réservé aux hommes. Ainsi, la possibilité pour le nom masculin de renvoyer à un référent /femelle/ est immédiatement présentée comme liée à une transgression des normes sociales de genre. Le référent implicite du mot avocat demeure donc /mâle/.

Dans les dictionnaires contemporains (XXème-XXIème siècles) utilisés dans nos recherches, l'hésitation entre féminisation et emploi du masculin générique se fait cette fois explicite :

« AVOCAT, ATE, subst. Personne défendant les intérêts de quelqu'un ou de quelque chose; Personne qui, étant inscrite au barreau, fait profession de défendre devant les tribunaux, soit oralement, soit par écrit, l'honneur, la vie, la liberté et les intérêts des justiciables et à les éclairer de ses conseils. Le baron de Rastignac veut-il être avocat ? (Balzac, Le Père Goriot,1835, p. 122). Seuls ont droit au titre d'avocat les licenciés en droit qui sont régulièrement inscrits au tableau ou au siège du barreau d'une cour d'appel ou d'un tribunal de grande instance. Ils doivent faire suivre leur titre d'avocat de la mention de ce barreau (décr. 10 avr. 1954, préc., art. 5). Rem. 1. Quand il s’agit d'une femme inscrite au barreau, on emploie gén. dans les textes officiels la forme masc., la forme fém. avocate appartient à la lang. parlée [...] ; Par anal. Celui, celle qui défend quelqu'un (en public ou non) [...]. L'avocate des pécheurs. » (ATILF)

«AVOCAT. n. m. Celui qui fait profession de défendre des causes en justice. Avocat fameux, célèbre, éloquent. Savant avocat. Avocat à la Cour d'appel de Paris, à la Cour de Cassation. Plaider par avocat. Votre avocat a bien plaidé. Avocat plaidant, Celui qui s'adonne principalement à la plaidoirie. [...] Il se dit figurément de Celui qui intercède pour un autre, qui en soutient, qui en défend les intérêts auprès de quelqu'un. Vous avez en lui un bon avocat. Je serai votre avocat auprès de lui. Il s'emploie aussi au féminin, Avocate. On compte maintenant d'assez nombreuses avocates au Palais. On dit encore Femme avocat. Fig., Sa mère fut son avocate » (AF8)

«AVOCAT, -ATE n. Auxiliaire de justice régulièrement inscrit à un barreau, et qui a pour profession d'assister ou de représenter les parties [...]. La nouvelle avocate a prêté serment. Avocat stagiaire. 
La conférence du stage des avocats. Admission dans l'ordre des avocats. Un avocat inscrit au barreau de Paris. Un avocat fameux, célèbre, éloquent. Une savante avocate » (AF9)

" Avocat, ate, n. Personne qui, régulièrement inscrite à un barreau, conseille en matière juridique ou contentieuse, assiste et représente ses clients en justice. (rem. Si le féminin avocate est désormais courant, on dit aussi avocat en parlant d'une femme.) Maître $X$, avocat. Consulter un avocat, un avocat-conseil. Prendre un avocat. Avocat plaidant, avocat à la Cour ; Fig. Avocat, avocate de : personne qui défend (une cause, une personne) » (PR15)

"AVOCAT, ATE, n.m. et n.f. Personne dont la profession est de plaider en justice. Avocat plaidant. Avocat consultant. Avocat du roi, avocat de la république, avocat impérial. Avocat général. Fig. Intercesseur. Il ne faut pas se faire l'avocat de l'injustice. » (NLIT)

« Avocat, avocate. n. Auxiliaire de justice dont la mission consiste à assister et à représenter en justice une personne qui se présente à lui et à défendre ses intérêts devant les différentes juridictions ; Personne qui intercède pour une autre ou lui fournit un moyen de défense : Se faire l'avocat d'une mauvaise cause. » (LAR)

L'ouverture progressive (à partir de la toute fin du XIXème ${ }^{21}$ ) de la profession aux femmes fait que la forme féminine du mot se banalise peu à peu, et surtout, est resémantisée (la forme n'ayant, au figuré, jamais cessé d’exister). Elle apparaît donc en vedette, du moins sous sa forme affixale (ate), dans tous les dictionnaires contemporains, sauf l'AF8 (le plus ancien). Toutefois, on peut d'emblée noter la persistance de certains phénomènes :

1. le maintien d'une possibilité de (voire d'une préférence pour) l'emploi du masculin : «Quand il s'agit d'une femme inscrite au barreau, on emploie gén. dans les textes officiels la forme masc. » (ATILF) ; «On dit encore Femme avocat » (AF8) ; « on dit aussi avocat en parlant d'une femme. » (PR15) ;

2. le lien explicite entre emploi de l'une ou de l'autre forme, et représentativité du type de référent visé vis-à-vis de la catégorie professionnelle en question : « Il s’emploie aussi au féminin, Avocate. On compte maintenant d'assez nombreuses avocates au Palais. On dit encore Femme avocat » (AF8). La légitimité de l'emploi du féminin pour désigner les référents /femelles/ appartenant à la catégorie /avocat, avocate/ serait d'abord liée à leur plus ou moins grande présence au sein de cette catégorie. Il ne suffit pas qu'un référent /femelle/ soit visé pour que le substantif soit actualisé au féminin, il faut aussi que ce type de référent soit considéré comme pertinent pour la catégorie professionnelle concernée ;

3. tous les exemples proposés en lien avec la catégorie professionnelle font apparaître le masculin, sauf trois : " On compte maintenant d'assez nombreuses avocates au Palais » (AF8) et « La nouvelle avocate a prêté serment [...]. Une savante avocate » (AF9).

On se retrouve donc, au terme de ce parcours lexicographique, avec un substantif qui se rencontre sous les deux formes dès le XVIème siècle, mais dont l'une se spécialise dans un emploi figuré du mot (la forme féminine), et dont l'autre a le monopole de la dénomination professionnelle (la forme masculine). Une fois que la profession s'ouvre, la forme féminine est rétablie dans le domaine non-figuré, mais la forme masculine demeure majoritaire. Une évolution de ce type met sur la voie d'une compréhension problématisée de la répartition en genre des noms de personne, et de la valeur dite "générique " du masculin, qui semble plutôt s'apparenter à un emploi culturellement motivé d'un genre plutôt que de l'autre. En 
contexte non-spécifique (et parfois même en contexte spécifique), on privilégierait le genre grammatical associé au sexe jugé représentatif d’une catégorie. L’idée est la suivante : dans le cadre de la dénomination de la personne, pour chaque catégorie visée par un radical substantival (identique pour avocat et avocate), émergerait généralement un type de référent privilégié, /mâle/ ou /femelle/, déterminé socialement, culturellement et historiquement (donc toujours potentiellement soumis à des évolutions). Ainsi, ce qui est traditionnellement compris comme une capacité à s’abstraire de la référence au sexe correspondrait en fait simplement à l'invisibilisation d'un type de référent au profit de l'autre. La sous-catégorie référentielle dominante ou sur-représentée serait donc mise en évidence par le choix des morphèmes de genre grammatical masculins (pour un prototype /mâle/) ou féminins (pour un prototype /femelle/). Ainsi la plus forte présence des masculins à valeur dite " générique » (on pourrait plutôt parler de "valeur culturelle »), en particulier dans le domaine des noms de métiers, semble directement liée au fait que les référents /mâles/ sont encore jugés représentatifs d'un grand nombre de catégories professionnelles. Ici, l'explication socio-culturelle et l'explication linguistique se recoupent pleinement.

\subsection{Au-delà du « sens référentiel »}

La « valeur générique » peut donc être largement questionnée par sa mise en perspective diachronique, et avec elle, les deux autres fondements de la prédominance du genre grammatical masculin en langue française : l'accord au masculin ne se défend que si, dans le cas de dénominations de la personne, le genre grammatical masculin peut renvoyer indifféremment aux deux types de référents ; le masculin forme première repose sur l'idée que le mot de départ, celui qui permet de dénommer avant toute caractérisation, est le mot masculin. Une fois ces questionnements amorcés, il semble nécessaire de s'intéresser d'un peu plus près à la description sémantique du genre grammatical lui-même. Considérant les constats posés ci-dessus, celle-ci ne semble plus pouvoir se réduire aux simples traits [+ mâle] et [+ femelle], qui reposent, comme nous l'avons vu, sur l'idée d'une motivation absolue entre sexe et genre grammatical des $\mathrm{NH}$ variables. Et en effet, on ne peut comprendre les phénomènes exposés ci-dessus en s'en tenant à ces deux traits sémantiques : la «valeur générique » constitue une incohérence logique si l'on considère que les morphèmes grammaticaux de genre masculin indiquent le renvoi à un/des référent(s) /mâle(s)/ ; on ne peut de plus, en se limitant à cette description, comprendre les différences de traitement sémantique entre les formes avocat et avocate que comme un surplus sémantique instable. Selon l'hypothèse que nous proposons, au genre grammatical masculin ne correspondrait donc pas un renvoi à la sous-catégorie référentielle $/ \mathrm{mâle} /$, mais plutôt une représentation sociale de ce qu'est le masculin, donc un ensemble de référents dits «masculins »; et de la même façon, au genre grammatical féminin correspondrait une représentation sociale de ce qu'est le féminin, donc un ensemble de référents dits « féminins ».

Cette proposition permet d'intégrer les données socio-culturelles et historiques à la description sémantique du genre grammatical, et non plus seulement de s'appuyer sur celles-ci pour en comprendre les fondements. Le trait sémantique associé au genre grammatical ne serait donc pas le trait [+ mâle] pour le masculin ou le trait [+ femelle] pour le féminin, mais plutôt le trait [+ stéréotypes associés au groupe social homme] pour le masculin ou le trait [+ stéréotypes associés au groupe social femme] pour le féminin. Nous entendons ici le terme stéréotype par distinction (mais non opposition) vis-à-vis de la 
notion de prototype évoquée plus haut, et reprenons en ce sens l’idée de «stéréotype partagé », développée par Georges Kleiber, qui repose sur l'existence de " connaissances qu'on croit être celles qui sont l'apanage des gens en général » (Kleiber 1999, p. 73). Il s'agit donc de «conventions sociales » - et non de " principes psychologiques » (idem) sur lesquelles repose la délimitation (établie, mais jamais définitive) des groupes sociaux évoqués. Ainsi, le fait de penser en termes de stéréotypes et de groupes sociaux plutôt que de catégories référentielles permet de remodeler la bipartition que constitue traditionnellement le genre grammatical au profit d'une répartition souple, et de sortir de ce que C. Michard nomme très justement une "sémantique référentielle a-sociologique » (Michard 2002, p. 135). Le genre grammatical, selon notre hypothèse, ne serait en rien porteur d’un sème unique et répétitif, fermé sur la référence au sexe, mais plutôt d'un ensemble de potentialités sémantiques, dont différents aspects peuvent être réalisés ou non selon le contexte et selon le mot étudié. Les réalisations sémantiques du genre grammatical, bien que stables, connaîtraient différentes actualisations possibles, variables en synchronie comme en diachronie, selon le radical substantival, le contexte d'énonciation, et les évolutions socio-culturelles. Cette idée d'un sens ouvert met sur la voie d'un questionnement du lien entre sexe et genre grammatical, et permet déjà en partie de sortir de la «perpétuation [en langue] d'une vision réifiée et statique des sexes, source d'inégalités et de discrimination » (Chetcuti \& Greco 2011, p. 11). Un des principaux effets de cette hypothèse est que, en limitant l'idée de motivation transparente qui sous-tend la conception traditionnelle du genre grammatical, elle permet en partie d'aplanir le rapport entre ses deux manifestations, et ce faisant de le dénaturaliser. En effet, avec le sens [+ stéréotypes associés au groupe social homme/femme], les descriptions sémantiques des deux genres grammaticaux sont présentées comme symétriques: le déséquilibre usuellement posé comme intrinsèque à la catégorie se situe en fait au niveau des stéréotypes eux-mêmes, mais en aucun cas au niveau de la catégorie grammaticale, et encore moins de quelconques catégories référentielles.

Il faut toutefois noter que, même avec la lecture sémantique - soutenue par l'analyse diachronique - que nous proposons, le stéréotype principal associé aux groupes hommes et femmes demeure encore aujourd'hui l'appartenance à la sous-catégorie référentielle /mâle/ ou /femelle/. Cependant, cette appartenance, conformément à la conception du stéréotype évoquée ci-dessus, n'est plus ni un critère nécessaire, ni un critère suffisant, et ne peut être considérée que comme une caractéristique majoritaire qui ne détermine nullement l'inclusion à ou l'exclusion de l'un ou l'autre groupe social.

\section{Conclusion}

L'introduction d'une pensée historicisante dans les études grammaticales sur le genre facilite très largement la mise au jour des mécanismes de stabilisation des croyances liées à cette catégorie. Elle permet ce faisant de visibiliser la construction d'un rapport hiérarchique, posant le genre grammatical masculin comme (pré-)dominant et capable de toujours «l'emporter » sur le féminin. La description sémantique que nous proposons, et qui découle de ce questionnement diachronique et synchronique, bien qu'encore imparfaite, permettrait à terme d'intégrer l'idée que la référence au sexe, dans le cadre de la dénomination de la personne, n’est ni nécessaire, ni automatique, même lorsque le renvoi à l'un des deux groupes homme ou femme $e^{22}$ est explicite. De ce fait, elle peut déjà être un recours en vue de la sensibilisation à certaines problématiques linguistiques et socio- 
linguistiques liées au genre grammatical, et souvent effacées par le caractère majoritairement décontextualisant des grammaires descriptives synchroniques.

\section{Références bibliographiques}

Académie française (2014). La féminisation des noms de métiers, fonctions, grades ou titres. Lien : http://www.academie-francaise.fr/actualites/la-feminisation-des-noms-de-metiers-fonctionsgrades-ou-titres-mise-au-point-de-lacademie

Arnauld, A. et Lancelot, Cl. (1660). Grammaire generale et raisonnée. Paris : Pierre le Petit.

ATILF. Trésor de la langue française informatisé. Lien : http://www.cnrtl.fr/lexicographie/

Auroux, S. (dir.) (1992) Histoire des idées linguistiques. Liège : Mardaga

Beauzée, N. (1819 (1767)). Grammaire générale. Paris : Auguste Delalain.

Berlion, D. (2105). Le Bled Grammaire. Paris : Hachette Éducation.

Bescherelle, L.-N. (1856). Dictionnaire national ou dictionnaire universel de la langue française. Paris : Garnier Frères.

Bescherelle, L.-N. et Bescherelle, H. (1838). Réfutation complète de la Grammaire de MM. Noël et Chapsal.

— (1864 (1835-36)). Grammaire nationale. Paris : Garnier Frères.

Bescherelle, L.-N. Et Lamotte, L. (1841). Grammaire de l'Académie. Paris : Delloye.

Bescherelle, L.-M. (1997). L'orthographe pour tous. Paris : Hatier.

Butler, J. (1990). Gender Trouble, feminisme and the subversion of identity. New York : Routledge.

- (2012). Postface. Dans Chetcuti, N. et Greco, L. La face cachée du genre. Paris: Presses Sorbonne Nouvelle.

Chapsal, C.-P. et Noël, F.-J.-M. (1845 (1823)). Nouvelle grammaire française. Paris : Hachette.

Chatard, A., Guimond, S. et Martinot, D. (2005). Impact de la féminisation lexicale des professions sur l'auto-efficacité des élèves : une remise en cause de l'universalisme masculin ? L'année psychologique, 2, vol.105, 249-272.

Chevalier, J-C. (1967). La grammaire générale de Port-Royal et la critique moderne. Langages, 7, 1633.

Chevalier, Y. (2013). Approches linguistiques du genre (gender). La clé des langues, lien: http://cle.ens-lyon.fr/plurilangues/approches-linguistiques-du-genre-gender--214114.kjsp

Damourette J. et Pichon, É. (1911-40). Des mots à la pensée : essai de grammaire de la langue française, Paris : D’Artrey

Delphy, C. (1977). The Main Ennemy. London : W.R.R.C.P.

Denis, D. et Sancier-Château, A. (1994). Grammaire du français. Paris : Le Livre de Poche.

Dictionnaire de l'Académie françoise (1694). Paris : Coignard.

Dictionnaire de l'Académie françoise (1740). Troisième édition. Paris : Coignard.

Dictionnaire de l'Académie françois (1798). Cinquième édition. Paris : Smits.

Dictionnaire de l'Académie française (1932-35). Huitième édition, version informatisée. Lien : http://www.cnrtl.fr/definition/academie8/couturier

Dictionnaire de l'Académie française. Neuvième édition, version informatisée. Lien : http://atilf.atilf.fr/academie9.htm

Dictionnaire Français en ligne. Larousse. Lien : http://www.larousse.fr/dictionnaires/francaismonolingue

Dorlin, E. (2007). Le Queer est un matérialisme. Femmes, genre, féminisme. Paris : Syllepse.

Durand, M. (1936). Le genre grammatical en français parlé, à Paris et dans la région parisienne. Paris : Bibliothèque du français moderne.

Elmiger, D. (2008). La féminisation de la langue en français et en allemand (querelle entre spécialistes et réception par le grand public). Paris : Honoré Champion.

Favre de Vaugelas, C. (1647). Remarques sur la langue française. Paris : Didot.

Fausto-Sterling, A. (2013). Les cinq sexes. Pouruqoi mâle et femelle ne sont

pas suffisants. Paris : Payot, traduit de l'anglais par Anne-Emmanuelle Boterf. 
Féraud, J.-F. (1787). Dictionnaire critique de la langue française. Marseille : Mossy.

Furetière, A. (1690). Dictionnaire universel. La Haye-Rotterdam : Arnout \& Reinier Leers.

Guillaumin, C. (1992). Sexe, race et pratique du pouvoir, l'idée de nature. Paris : Côté-femmes.

Guillon, J. (1990). La grammaire pour tous. Paris : Hatier.

Khaznadar, E. (1990). Le nom de la femme, virtualisation idéologique et réalité linguistique (thèse de doctorat sous la direction d'A. Borillo). Toulous : Université de Toulouse-Le Mirail.

- (2002). Le féminin à la française, académisme et langue française. Paris : L’Harmattan.

- (2007). L'homme générique... dans les savanes de la préhistoire. Langage et société, 119, 131155.

- (2008). Sexisme et grammaires scolaires. Langue-fr.net. Lien : http://www.langue-fr.net/Sexismeet-grammaires-scolaires

Kleiber, G. (1999). La sémantique du prototype. Paris : Presses universitaires de France.

Larousse, P. (1886). Nouveau dictionnaire illustré français. Paris : Larousse \& Boyer.

Lhomond, C.-F. (1780). Élémens de la grammaire françoise. Paris : Colas.

Martinet, A. (1956). Le genre féminin en indo-européen : examen fonctionnel du problème. Bulletin de la Société de linguistique de Paris, 52, 83-95.

- (1996). Genre et sexe. Histoire et grammaire du sens (dir . Auroux, S. et Meschonnic, H.), 213218. Paris : Armand Colin.

Mathieu, N-C. (1991). L'anatomie politique. Catégorisations et idéologies du sexe. Paris: Côtéfemmes.

Maupas, C. (1632). Grammaire et syntaxe françoise. Rouen : Jacques Cailiove.

Meigret, L. (1550). Le tretté de la grammere françoeze. Paris : Chrestien Wechel

Michard, C. (1996). Genre et sexe en linguistique : les analyses du masculin générique. Mots, 49, 2947.

- (1999). Humain/ femelle : deux poids deux mesures dans la catégorisation de sexe en français. Nouvelles questions féministes, 1, vol. 20, 53-95.

- (2002). Le sexe en linguistique. Sémantique ou zoologie ? Paris : L’Harmattan.

Meillet, A. (1921). Linguistique historique et linguistique générale. Paris : Honoré Champion.

Mok, Q. I. M. (1968). Contribution à l'étude des catégories morphologiques du genre et du nombre dans le français parlé actuel. Paris : Mouton.

Oudin, A. (1972 (1632)). Grammaire françoise rapportée au langage du temps. Genève : Slatkine Reprints.

Puig de la Bellacasa, M. (2003). Divergences solidaires. autour des politiques féministes des savoirs situés. Multitudes, 12, 39-47.

Ramée (de la), P. (1572). Grammaire. Paris : André Wechel.

Rey, A. et J. Rey-Debove (dir.) (1985), Le petit Robert 1, dictionnaire alphabétique et analogique de la langue française, Paris : Le Robert.

- (2015), Le petit Robert 1, dictionnaire alphabétique et analogique de la langue française, Paris : Le Robert.

Richelet, P. (1680). Dictionnaire françois, contenant les mots et les choses. Genève : Widerhold.

Riegel, M., Pellat, J.-C. et Rioul, R. (2011). Grammaire méthodique du français. Paris : Presses universitaires de France.

Steinberg , S. (2010). Hiérarchies dans l’ancien régime. Dans Riot-Sarcey, M. (dir.). De la différence des sexes. Le genre en histoire. Paris : Bibliothèque historique Larousse.

Viennot, É. (2014). Non, le masculin ne l'emporte pas sur le féminin !, petite histoire des résistances de la langue française. Donnemarie-Dontilly : iXe. 
${ }^{1}$ Nous désignons par «non-anthropomorphisés » des animés qui, bien que sexués, ne sont pas différenciés en fonction de ce critère, car ne présentent pas d'utilité immédiate pour l'être humain, et n’entretiennent pas de rapport privilégié avec lui.

${ }^{2}$ Classe des noms d'humains au sein de laquelle nous comprenons les noms d'animés anthropomorphisés comme des cas limites. Voir, au sujet de cette classe, les travaux du groupe de recherche NHUMA.

${ }^{3}$ Cette étude sera centrée sur les pratiques grammaticales françaises et non francophones, la diversité de ces dernières étant très clairement liée à des divergences dans les politiques linguistiques officielles et dans les engagements militants. Une étude sur les pratiques québécoises ou sénégalaises du genre grammatical, malgré une langue commune, ne s'appuierait pas sur les mêmes constats de départ qu'une étude sur les pratiques françaises. La perspective historique adoptée dans ce papier justifie donc de se concentrer sur un univers de croyances délimité.

${ }^{4}$ les piliers sont quasi systématiquement présents dans les ouvrages grammaticaux descriptifs ainsi que dans les travaux non-spécialisés ou simplement ne s'attardant pas sur la question du genre grammatical. Toutefois, il faut d'emblée noter que de nombreux travaux proposent un questionnement critique de ces trois règles, dans des cadres théoriques très variés. L'accord au masculin et la «valeur générique " sont généralement questionnés dans des études syntaxiques et/ou sémantiques: D. Elmiger rappelle que les entreprises de féminisation menées par les militantes et chercheuses féministes ont en partie pour but de « provoquer une prise de conscience par rapport au masculin et à sa généricité » (Elmiger 2008, p.143) ; les travaux de C. Michard, sur lesquels nous reviendrons, examinent les fondements possibles de la hiérarchisation entre les genres (Michard 1996 sur le masculin " générique », Michard 1999 sur l'asymétrie entre les genres grammaticaux, Michard 2003 sur l'histoire des théories linguistiques intégrant la question du genre grammatical, etc.) ; certaines expériences psycho-cognitives tendent à montrer que la primauté du masculin en langue française a des effets réels sur l'auto-évaluation des femmes comme des hommes (Chatard, Guimond et Martinot 2005), etc. Le masculin forme première a été vivement remis en cause dans des recherches phonologiques, centrées sur la langue parlée ainsi que sur les notions de moindre effort et d'efficacité descriptive (selon Q.I. Mok, la forme féminine constituerait un meilleur point de départ pour la description des formes variables en genre, et notamment des adjectifs, du fait de la plus forte prédictibilité des phonèmes masculins à partir des phonèmes féminins (Mok 1968)). E. Khaznadar a cherché dans ses travaux à bousculer les conceptions du genre grammatical sur les trois fronts, en questionnant notamment l'héritage des théories structuralistes de la marque (Khaznadar 1990), ainsi que les discours de validation de la supériorité du genre grammatical masculin (Khaznadar 2002, 2008) et d'invisibilisation des femmes (Khaznadar 2007). Sans pour autant les oublier, nous ne nous attarderons pas, en ce début de parcours, sur ces travaux de recherche, et nous nous centrerons plutôt sur les définitions non-critiques du genre grammatical proposées par les usuels.

5 Les questionnements sur la naturalisation ont d'ailleurs permis de repenser les sexes euxmêmes, en les sortant d'un cadre nécessairement dual et en s'intéressant à la diversité de leurs réalisations biologiques et physiologiques (Fausto-Sterling 2013).

$6 \quad$ Dans les autres cas, les dictionnaires présentent soit les formes masculine et féminine dans des entrées séparées (c'est par exemple le cas du NLIT pour boucher et bouchère), les traitant comme des homonymes, soit uniquement la forme masculine, en attestant (AF8) ou non la forme féminine dans le corps de l'article. Les substantifs en question ne sont donc plus présentés comme variables.

${ }^{7}$ Les seuls arguments pouvant justifier et expliquer ces pratiques sont l'argument économique du gain de place pour les dictionnaires imprimés, et celui du gain de temps pour les versions imprimées et numériques.

${ }^{8}$ On pourrait aussi discuter la simplification extrême qui sous-tend l'énoncé «L’héritage latin a opté pour le masculin », en rappelant d'une part que la langue latine n'est pas l'unique matrice du 
français, et d'autre part qu'un nombre conséquent de neutres latins ayant un pluriel en - $a$ sont passés au féminin en français (folium, granum, etc.), par des phénomènes d'analogie.

${ }^{9}$ Les emplois dits " génériques » recouvrent en linguistique tous les emplois non-spécifiques des substantifs, actualisés par des articles définis (emplois génériques stricts, emplois intentionnels) ou indéfinis (opérations d'extraction aléatoire, opérations de classification).

${ }^{10}$ André Martinet est un des auteurs majeurs, dans la lignée d'Antoine Meillet, ayant proposé une explication historique à ce fonctionnement du genre grammatical masculin. Son hypothèse, développée dans «Le genre féminin en indo-européen » (Martinet 1956) fait du genre grammatical féminin le genre originellement oppositif, apparu pour indiquer une différenciation d'avec le genre commun préalable, dont hériterait le masculin. On trouve dans ce récit des origines un des fondements possibles des théories de la marque : il n'y aurait pas de symétrie possible entre les deux genres grammaticaux en langue française. Cette hypothèse extrêmement séduisante peut pourtant être critiquée comme régression téléologique à partir des théories de la marque.

11 Ces processus de féminisation, bien que politiquement pertinents, demeurent cependant théoriquement critiquables, car fondés sur un principe de différenciation binaire potentiellement essentialisant.

${ }^{12}$ https://en.wikipedia.org/wiki/User:Anthere/Discussion_language_non_sexiste

${ }^{13}$ http://www.lefigaro.fr/livres/2014/10/15/03005-20141015ARTFIG00163-feminisation-desnoms-la-mise-au-point-de-l-academie-francaise.php

${ }^{14}$ http://information.tv5monde.com/terriennes/feminisation-des-mots-la-france-en-retard-22877

${ }^{15}$ La théorie développée par J. Butler repose sur l'idée que les discours et pratiques genrés produisent le genre, et ce dès la première assignation par le corps médical (à la catégorie « fille » ou " garçon »), puis tout au long de l'existence : " [Le genre] n'agit pas par lui-même mais devient actif dans et à travers divers modes d'adresse, dans les situations où l'on est nommé, à travers les modes de référence, les gestes et les mouvements corporels, dans des documents juridiques, psychiatriques ou médicaux, dans divers médias ou discours publics » (Butler 2012, p. 148).

${ }^{16}$ Il existe bien sûr des productions grammaticales avant cette date en France (notamment sur le et en provençal), mais les entreprises linguistiques de François Ier, évidemment liées à son projet politique qui marque les débuts de l'absolutisme, imposent progressivement «la suprématie du francien sur les autres parlers régionaux » (Auroux 1992, p. 360).

${ }^{17}$ Cette prise en considération est un des apports majeurs de la Grammaire d'Arnauld et Lancelot, qui sort en partie du formalisme du XVIIème siècle. Voir à ce propos l'article de Jean-Claude Chevalier sur l'ouvrage d'Arnauld et Lancelot (Chevalier 1967).

${ }^{18}$ Plus précisément, Vaugelas s’intéresse au parler courtisan de son temps, validé par les auteurs de son temps.

${ }^{19}$ Les premières demandes de financement public de manuels scolaires ont lieu dès les années 1790, et ce mouvement est entériné au XIXème siècle, notamment par la loi Guizot de 1830 sur l'instruction publique et la liberté d'enseignement. Ainsi, les règles de grammairiens, réservées jusqu'ici à une certaine frange de la population, se généralisent et se banalisent progressivement par le biais des ouvrages grammaticaux destinés à l'enseignement.

${ }^{20}$ Cette idée sera parachevée moins d'un siècle plus tard par la théorie de la « sexuisemblance » développée par Damourette et Pichon (1911-40), qui élargit l'idée de motivation entre sexe et genre à tous les noms (et non plus seulement aux $\mathrm{NH}$ ), et qui trouve peut-être une de ses sources directes dans les travaux des frères Bescherelle, selon qui «l'attribution du genre aux êtres dépourvus de sexe fut [...] une véritable métaphore » (Bescherelle 1838, p.18). 
21 En 1900, Olga Balachowsky-Petit et Jeanne Chauvin deviennent les premières avocates françaises.

22 Groupes eux-mêmes évidemment discutables et souvent soumis à des processus de naturalisation et/ou de psychologisation. 